\title{
Advanced Patient or Elder Fall Detection based on Movement and Sound Data
}

\author{
Charalampos Doukas \\ Dep. of Information \& Communication Systems \\ Engineering \\ University of the Aegean \\ Samos, Greece \\ doukas@aegean.gr
}

\author{
Ilias Maglogiannis \\ Dep. of Information \& Communication Systems \\ Engineering \\ University of the Aegean \\ Samos, Greece \\ imaglo@aegean.gr
}

\begin{abstract}
The paper presents am initial implementation of a patient monitoring system that may be used for patient activity recognition and emergency treatment in case a patient or an elder falls. Sensors equipped with accelerometers and microphones are attached on the body of the patients and transmit patient movement and sound data wirelessly to the monitoring unit. Applying Short Time Fourier Transform (STFT) and spectrogram analysis on sounds detection of fall incidents is possible. The classification of the sound and movement data is performed using Support Vector Machines. Evaluation results indicate the high accuracy and the effectiveness of the proposed implementation.
\end{abstract}

Keywords-component; patient monitoring, fall detection, movement and sound analysis, SVM classification

\section{INTRODUCTION}

The telemonitoring of human physiological data, in both normal and abnormal situations of activity, is interesting for the purpose of emergency event detection or long term datastorage for later diagnosis or for the purpose of medical exploration. In the case of elderly people living on their own, there is a particular need for monitoring their behavior. The first goal of this surveillance is the detection of major incidents such as a fall, or a long period of inactivity in a part of their area. The early detection of fall is an important step to alert and protect the subject, so that serious injury can be avoided. Fall detection is an important part of human body movement analysis; it is considered as an area of increasing importance and interest to practitioners, researchers, and health industry and most importantly, it is vital for indication of emergency cases. Motion data acquired from accelerometers and audiovisual content from the patient's environment have been proposed as practical, inexpensive and reliable methods for monitoring ambulatory motion in elderly subjects for the detection and prediction of falls. Robust classification of the aforementioned data enable the development of more reliable methods for monitoring long term change in physiological indicators such as parameters of gait, balance, energy expenditure and general well-being.

This paper presents the initial implementation of a patient fall detection platform based on accelerometer and sound data. Body sensors collect the movement data and microphones

Permission to make digital or hard copies of all or part of this work for personal or classroom use is granted without fee provided that copies are not made or distributed for profit or commercial advantag and that copies bear this notice and the full citation on the first page. To copy otherwise, to republish, to post on servers or to redistribute to lists, requires prior specific permission and/or a fee. PERVASIVEHEALTH 2008, 30 Jan - 1 Feb. Tampere, Finland

Copyright $\odot 2008$ ICST 978-963-9799-15-8

DOI 10.4108/ICST.PERVASIVEHEALTH2008.2520 capture environment sounds and transmit them wirelessly to the monitoring unit. Appropriate data classification using Support Vector Machines [19], can classify the recorded data and distinguish falls from related events (e.g., walk, run, fall of objects, etc.). The rest of the paper is organized as follows; Section 2 discusses related work in the context of patient activity and fall detection. Section 3 describes the acquisition of the patient movement data using sensors, whereas Section 4 presents the proposed sound processing method. Section 5 discusses the data classification using Support Vector Machines, while Section 6 presents the evaluation results and Section 7 concludes the paper.

\section{RELATED WORK AND SYSTEM ARCHITECTURE}

Although the concept of patient activity recognition with focus on fall detection is relatively new, there exists significant related research work, which may be retrieved from the literature ([1]-[16]). Information regarding the patient movement and activity is frequently acquired through visual tracking of the patient's position. In [6] and [15] overhead tracking through cameras provides the movement trajectory of the patient and gives information about user activity on predetermined monitored areas. Unusual inactivity (e.g., continuous tracking of the patient on the floor) is interpreted as a fall. Similarly, in [10] omni-camera images are used in order to determine the horizontal placement of the patient's silhouettes on the floor (case of fall). Success rate for fall detection is declared at $81 \%$ for the latter work. Head tracking is used in [13] in order to follow patient's movement trajectory with a success rate of fall detection at $66.67 \%$. Environmental sound processing is used in [24]-[26] for monitoring the overall behavior of patients and detection of alarm incidents.

The aforementioned activities detection methods based on audio and visual information of the user require capturing equipment and thus are limited to indoor environment usage. In addition, some of the methods require also the a-priori knowledge of the area structure (e.g., obstacles, definition of floor, etc.), or user information (e.g., height in [10]). A different approach for collecting patient activity information is the use of sensors that integrate devices like accelerometers, gyroscopes, contact sensors and microphones. The decrease of sensors size and weight, in conjunction with the introduction of 
embedded wireless transceivers allows their pervasive placement on patients and the transmission of the collected movement and audio information to monitoring units wirelessly. The latter approach is less depended on the patient and environmental information and can be used for a variety of applications for user activity recognition ([1], [3], [9]). Regarding fall detection, authors in [2], [7], [8], [12] use accelerometers, gyroscopes and tilt sensors for movement tracking. Collected data from the accelerometers (i.e., usually rotation angle or acceleration in the $\mathrm{X}, \mathrm{Y}$ and $\mathrm{Z}$ axis) is used in order to verify the placement of the patient and time occupation in rooms and detect abrupt movement that could be associated with fall. Detection is performed using predefined thresholds [1], [3], [4], [7] and association between current position, movement and acceleration [2], [8], [12]. Finally, area sensors have been used in order to track and analyze patient movement; authors in [11] describe a vibration-based detector that can detect falls based on the vibration caused on the floor. In [5] infrared sensors are used in order to provide thermal information regarding the patient's location and movement. The latter approaches do not require from the user to wear or carry sensor devices, however they demand more expensive equipment to be installed on the surrounding environment. Most of the related work based on accelerometers for fall detection, focuses on the elderly and may not be used for general classification of the patient movement activity or usage in younger ages (e.g. interpretation of running has not been assessed against falling). In addition, detection is usually performed through predefined thresholds and thus results can be depended to the movement patterns of the users.

Regarding fall detection using sound processing, most of the related work focuses on collecting and analyzing sound data captured from the patient's environment. The latter method needs special equipment to be installed on site and can be used exclusively in indoor environments. Additionally it requires much effort for isolating sounds generated within patient's proximity from other sounds. To our best knowledge, there is no other work within literature that incorporates sound capturing devices (i.e. microphones) on body sensor networks. The latter method has also the advantage of better distinguishing surrounding noise against sounds generated by a fall.

The proposed system may be used for a variety of patient activity recognition since it can successfully distinguish events between walk, run, object's fall and actual patient fall. In addition it is not biased by the movement pattern or the physiology of a specific patient (i.e. it can perform successfully with movement data from different individuals) and it does not apply restrictions to the user's environment (e.g., it can be used in outdoor environments as well). The system follows the architecture illustrated in Figure 1. Accelerometer and sound data are collected through the sensor attached on the user's foot and are transmitted wirelessly to the monitoring node. The data is properly transformed in a suitable format for the classifier and the classification phase begins. Based on a predefined classification model (i.e. train model), the patient status is detected (i.e. emergency status when fall detected, normal status otherwise). The basic modules of the proposed implementation are discussed in the following sections.

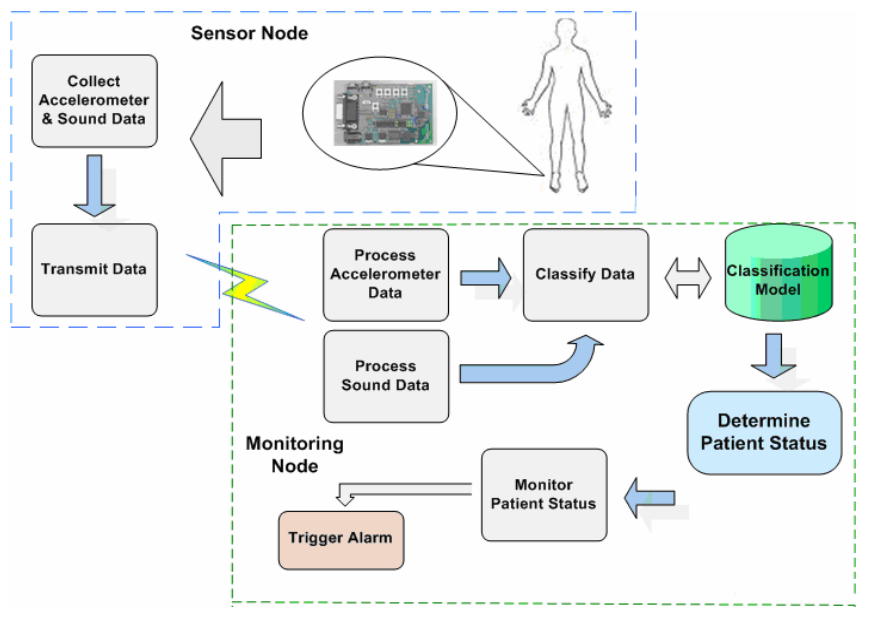

Figure 1. Platform Architecture and Data interaction between the sensor and monitoring node

\section{Patient Movement Data AcQuisition}

The MC13192 [2] sensor has been used in our system for data collection. The latter contains a $2.4 \mathrm{GHz}$ wireless data transceiver RF reference design with printed circuit antenna, which provides all hardware required for a complete wireless node using IEEE 802.15.4 (ZigBee) [17] packet structure. It includes an RS232 port for interface with a personal computer, background debug module for in-circuit hardware debug, four switches and LEDs for control and monitoring, a low-power, low-voltage MCU (MicroController Unit) with $60 \mathrm{~KB}$ of onchip Flash which allows the user flexibility in establishing wireless data networks, two 3D Accelerometers for X, Y and Z axis and one microphone. Figure 2 shows the SARD ZigBee node [18]. ZigBee has been chosen as communication technology for a number of reasons:

- Low cost and very low power consumption:

- Low complexity that makes the protocol ideal for integration on sensor nodes.

- Higher range compared to Bluetooth (up to 100 meter).

- Can be used for automatic creation of mesh networks and

- Contains built-in security measures.

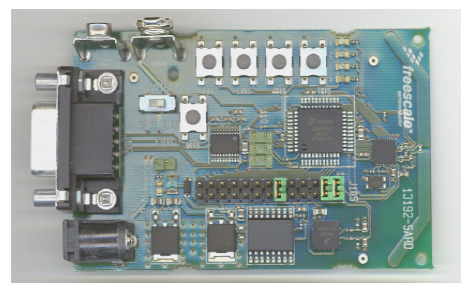

Figure 2. The SARD ZigBee node. The node acts as both receiver and transmitter. The RS232 interface provides connectivity with the monitoring device (e.g., a PDA) when the node is used as receiver. The transmitter is attached on user and sends data through the ZigBee wireless protocol.

The user's foot has been selected for acquiring movement data due to the fact the majority of human movements require the movement of the feet at one of the three axes (i.e. X, Y and $Z$ ). Thus the placement of the sensor on the foot allows the collection and association of accelerometer data with a wider 
range of human activity (e.g., walk, run, lie, etc.). The acquired data contain the sound signal and information about the patient's movement in the context of acceleration in the $\mathrm{X}, \mathrm{Y}$ and $\mathrm{Z}$ axis and are transmitted wirelessly to the monitoring node.

\section{PATIENT Sound Data ACQuisition And PRocessing}

The capture sound signal from the sensor's microphone is transmitted to the monitoring module for further processing. Related work within literature ([24]-[26]) uses Discrete Wavelet Transformation (DWT) for signal denoising and detection of special sounds that can be associated with a patient's state. However the latter transformation has high complexity and the results can be biased from the decomposition level of the wavelet coefficients. The presented work uses spectrogram analysis that is based on short-timeFourier Transform (STFT) on the signal for the detection of sounds generated by the fall of the human body. Given a signal $\mathrm{x}(\mathrm{t})$ and its Fourier Transform $\mathrm{X}(\tau, \omega)$ the STFT is:

$$
\operatorname{STFT}\{x()\} \equiv X(\tau, \omega)=\int_{-\infty}^{\infty} x(t) \omega(t-\tau) e^{-j \omega} d t,(\text { Equation 1) }
$$

The spectrogram is respectively given by the magnitude of the STFT of one function:

$$
\operatorname{spectrogram}(t, \omega)=|\operatorname{STFT}(t, \omega)|^{2}, \quad \text { (Equation 2) }
$$

In the most usual format of a spectrogram, the horizontal axis represents time, the vertical axis is frequency, and the intensity of each point in the image represents amplitude of a particular frequency at a particular time (see Figure 3). Based on conducted experiments the relative amplitude of a signal and the peak frequency at a given time can give a successful indication of a patient fall sound as captured by the on-body sensor microphone; Body falls generate low frequency sounds with high amplitude. Using a threshold of $>90 \%$ for relative signal amplitude and $<200 \mathrm{~Hz}$ for peak frequency, the differentiation of a fall sound against other sounds is possible. More precisely, over a series of 20 sound samples containing both body fall sounds and background noise (e.g., radio, object falls, etc) the detection of the fall was possible for the $80 \%$ of them. Different types of floor (i.e. wood, cement and flooring tile) were also used. The presented method has low computational complexity and can be easily integrated on sensor devices for real time sound processing. The unsuccessful detection of fall sounds was observed in cases where high amplitude sounds were generated in close proximity to the microphone. However, the correlation of the sound data with the movement data can provide much more accurate results as presented in the following section.

\section{DATA Classification USING SVM}

This Section provided information regarding the classification method used, parameters and data modeling. The Support Vector Machines (SVMs) is a popular algorithm for data classification into two classes [19], [20]. SVMs allow the expansion of the information provided by a training data set as a linear combination of a subset of the data in the training set (support vectors).
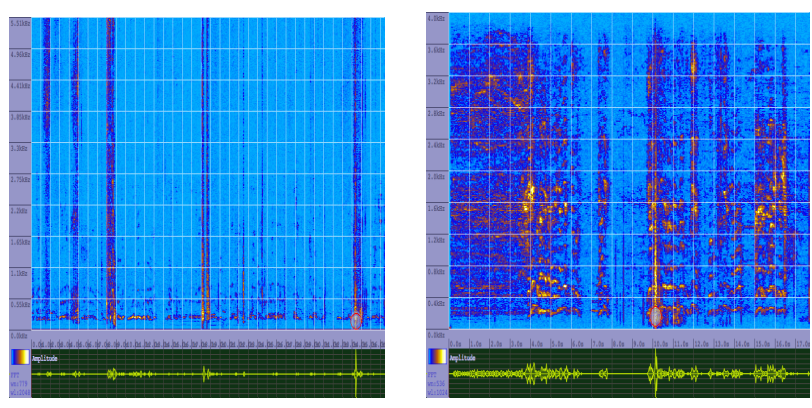

Figure 3. Spectrogram figures of two sample sound sequences. The X-axis represents time, the Y-axis the signal frequency, whereas each pixel color represents the amplitude intensity (blue $=$ high intensity, yellow-red $=$ high intensity). Sample (a) contains the sound signal generated by the fall of a heavy object and a patient body fall. Sample (b) contains background noise (radio) and a body fall sound. Spectrogram images are generated using Sonogram SW [27].

These vectors locate a hypersurface that separates the input data with a very good degree of generalization. The training vectors can be mapped into a higher dimensional space using different kernel functions (Linear, Polynomial, Radial Basis Function (RBF), Sigmoid). In order to make an efficient selection of the most suitable kernel type for the presented platform, the aforementioned kernel types have been validated against accelerometer and sound data. Three classification classes have been defined according to following cases; patient fall state, patient walk state and patient run state. A SVM train model has been created from the latter data using the tool presented in [21]. The input data has the following form:

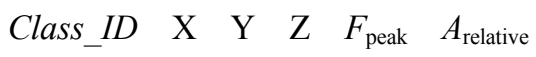

where Class_ID $\in[1,3]$ and represents the movement case (i.e. 1 for walk, 2 for run and 3 for fall), $X$ the acceleration value in the $\mathrm{X}$ axis, $\mathrm{Y}$ the acceleration value in $\mathrm{Y}$ axis and $\mathrm{Z}$ the acceleration value in $\mathrm{Z}$ axis respectively. $F_{\text {peak }}$ represents the frequency peak whereas $A_{\text {relative }}$ the relative signal amplitude at the current time. For simplicity, all values were normalized to $[0,1]$. The same data have been used in order to verify the accuracy of each kernel. Figure 4 illustrates the accuracy of each kernel type for different $\mathrm{C}$ parameter values. As results indicate, the RBF kernel behaves much better in terms of accuracy (approaches 98.2\%), and thus it has been selected for data classification.

\section{FAll Detection And Evaluation Results}

In order to evaluate the efficiency and accuracy of the presented platform in the context of detecting patient falls, three different experiments were conducted; two volunteers wearing the sensors devices described in Section 3 performed three combinations of movement types; a) simple walk, b) simple walk and fall, c) simple walk and run. During all three experiments various types of background noise (e.g., radio, heavy object falls, etc.) were also used in order to make the evaluation more realistic. The classification procedure was performed in two different modes; the first one includes movement classification using only the $\mathrm{X}, \mathrm{Y}$ and $\mathrm{Z}$ acceleration data, whereas the second one using the latter in addition to the 
collected sound features (i.e. the frequency peaks and related amplitudes).

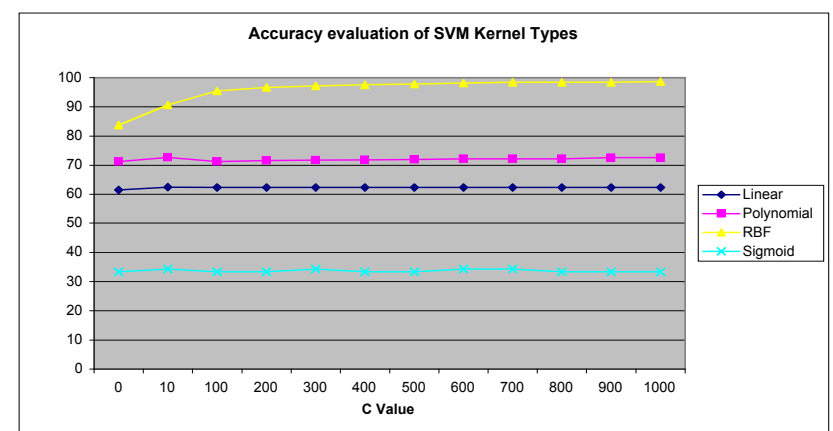

Figure 4. Accuracy Evaluation of different SVM Kernel types for different C values. $\mathrm{X}$ axis represents the $\mathrm{C}$ value range between $[1,1000]$ and the $\mathrm{Y}$ axis the accuracy percentage.

The classification model with the parameters and data described in Section 5 was used. A predefined period of 5 seconds was used as a time frame in order to feed real time acceleration and sound data to the classifier. The output was an estimation of the movement type. Based on the number of sequential occurrence of a specific movement type, decision regarding a patient fall is taken. In order to improve the accuracy of the latter decision, Kalman filtering [22], [23] has been applied on the sequence of the movement type association of each acceleration data set.

Figure 5 represents the classification results from the conducted experiments using the trained SVM model. Light colored lines represent original results whereas dark colored lines results after applying Kalman filtering. For annotation purposes, the three movement types were associated with three integers; 1 for walk, 2 for run and 3 for fall respectively. Actual run and fall events are also annotated on the diagrams. For each experiment two different diagrams were generated; one illustrating classification results based exclusively on acceleration data and one illustrating classification based on both acceleration and sound data.

As it is indicated, Kalman filtering improves the overall detection by smoothing the sequential occurrences of run or fall events respectively. In addition, the use of sound as additional classification feature has increased the accuracy of fall detections by minimizing the false ones in cases of simple walk (Figure 5 (b)) and of walk and run (Figure 5 (f)). A threshold $t$ $=10$ has been selected for determining the occurrence of a fall or run event from the total sequence of classified movement types (i.e. if sequential occurrence of fall movement types $>10$ then a fall is detected). Using the aforementioned classification and the latter threshold value, fall events were successfully detected in all cases, whereas run events were successfully detected at $96.72 \%$.

\section{CONCLUSIONS}

In this paper we presented the initial implementation of a system for detecting patient falls combining movement and sound data. User movement is monitored using sensor devices that provide information regarding the acceleration in the $\mathrm{X}, \mathrm{Y}$ and $\mathrm{Z}$ axis whereas sound is captured from a microphone embedded on the sensor. Proper data classification based on Support Vector Machines provides fall detection with satisfying accuracy. Future related work might include the enhancement of the platform with additional sound and video processing for the detection of emergency events based on patient's voice and exploiting computer vision techniques.

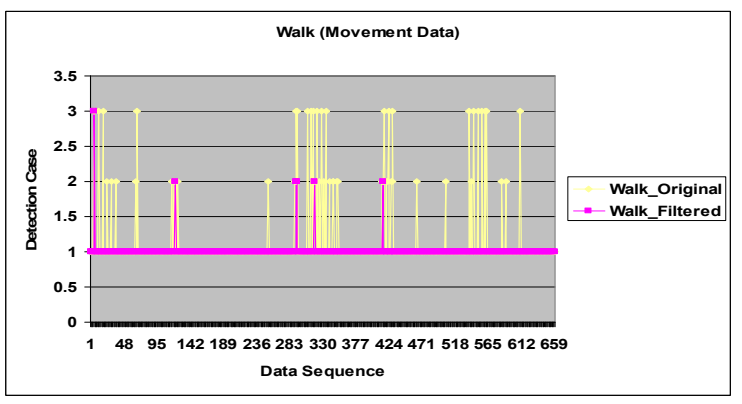

(a)

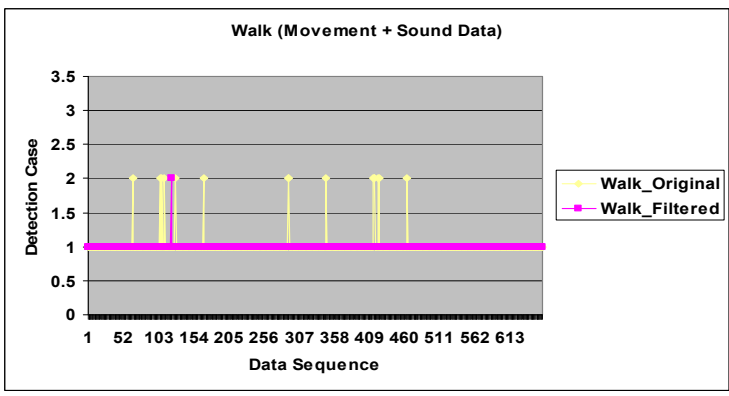

(b)

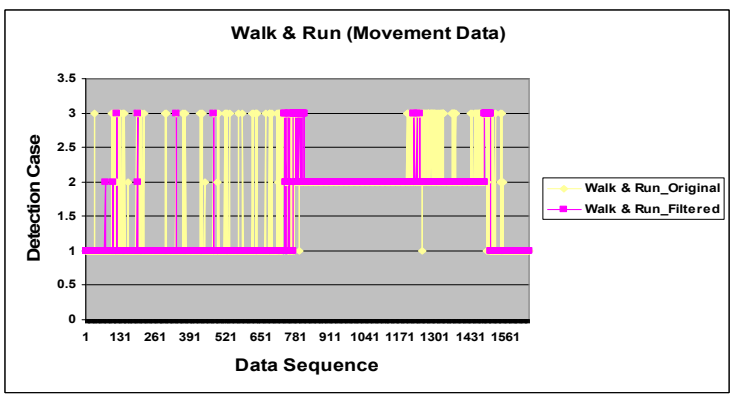

(c)

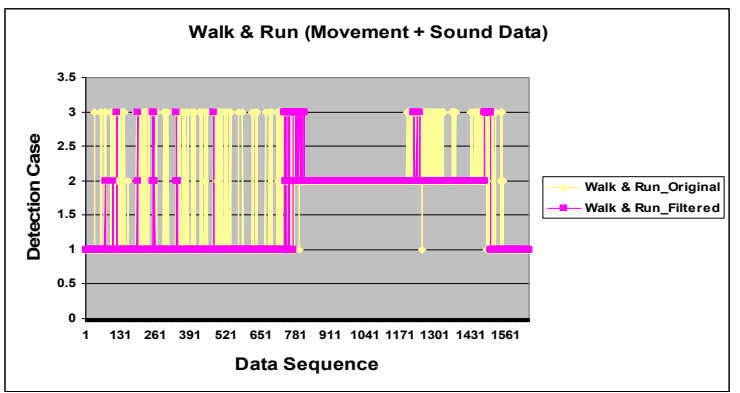

(d) 


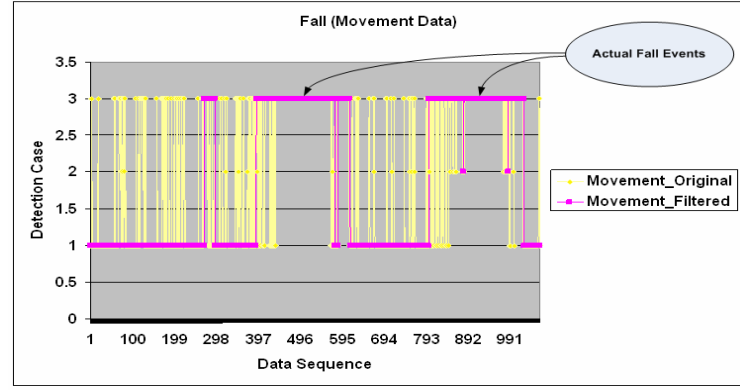

(e)

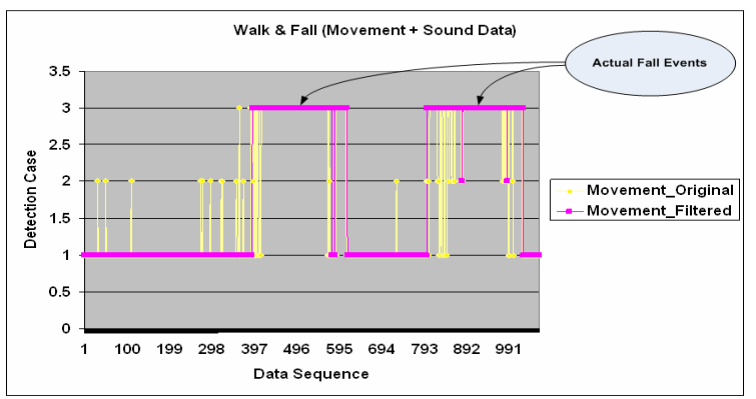

(f)

Figure 5. Classification results based on the SVM trained model for three different types of movement: (a) \& (b) simple walk, (c) \& (d) walk and fall and (e) \& (f) walk and fall. $\mathrm{X}$ axis represents the acceleration data sequence and $\mathrm{Y}$ axis the corresponding movement type (i.e. 1 for walk, 2 for run and 3 for fall). Light colored lines represent original results whereas dark colored lines results after applying Kalman filtering. Actual run and fall events are annotated on the diagrams.

\section{ACKNOWLEDGMENT}

This Research work is funded by the Community Initiative INTERREG III/A Greece - Cyprus under the Grant "Telemedicine Network for Remote Locations in the North Aegean Region".

\section{REFERENCES}

[1] Noury N., Herve T., Rialle V., Virone G., Mercier E., Morey G., Moro A., Porcheron T., "Monitoring behavior in home using a smart fall sensor and position sensors", In Proc. of 1st Annual International Conference on Microtechnologies in Medicine and Biology, pp. 607610, Oct. 2000.

[2] Noury N., "A smart sensor for the remote follow up of activity and fall detection of the elderly", In Proc. of 2nd Annual International Conference on Microtechnologies in Medicine and Biology, pp. 314317, May 2002.

[3] Prado M., Reina-Tosina J., Roa L., "Distributed intelligent architecture for falling detection and physical activity analysis in the elderly", In Proc. of the 24th Annual IEEE EMBS Conference, pp. 19101911, Oct. 2002.

[4] Fukaya K., "Fall detection sensor for fall protection airbag", In Proc. of the 41st SICE Annual Conference, pp. 419-420, Aug. 2002.

[5] Sixsmith A., Johnson N., "A smart sensor to detect the falls of the elderly", IEEE Pervasing Computing, Vol. 3, No. 2, pp. 42-47, AprilJune 2004.

[6] Nait-Charif, H. McKenna, S.J., "Activity summarisation and fall detection in a supportive home environment", In Proc. of the 17th International Conference on Pattern Recognition ICPR 2004, pp. 323236, Aug. 2004.

[7] Hwang, J.Y. Kang, J.M. Jang, Y.W. Kim, H.C., "Development of novel algorithm and real-time monitoring ambulatory system using Bluetooth module for fall detection in the elderly", In Proc. of the 26th
Annual International Conference of the IEEE Engineering in Medicine and Biology Society, pp. 2204-2207, 2004.

[8] Suhuai Luo, Qingmao Hu, "A dynamic motion pattern analysis approach to fall detection", In Proc. of the 2004 IEEE International Workshop on Biomedical Circuits and Systems, pp. 1-8a, Dec. 2004.

[9] Shuangquan Wang, Jie Yang, Ningjiang Chen, Xin Chen, Qinfeng Zhang, "Human activity recognition with user-free accelerometers in the sensor networks", In Proc. of International Conference on Neural Networks and Brain, pp. 1212-1217, Oct. 2005.

[10] S.-G. Miaou, Pei-Hsu Sung, Chia-Yuan Huang, "A Customized Human Fall Detection System Using Omni-Camera Images and Personal Information", In Proc. of 1st Transdisciplinary conference on Distributed Diagnosis and Home Healthcare, pp. 39-42, 2006.

[11] Alwan, M. Rajendran, P.J. Kell, S. Mack, D. Dalal, S. Wolfe, M. Felder, R. "A Smart and Passive Floor-Vibration Based Fall Detector for Elderly", In Proc. of 2nd Information and Communication Technologies Conference, ICTTA 06, pp. 1003-1007, April 2006.

[12] Allen, F.R. Ambikairajah, E. Lovell, N.H. Celler, B.G., "An Adapted Gaussian Mixture Model Approach to Accelerometry-Based Movement Classification Using Time-Domain Features", In Proc. of 28th Annual International Conference of the IEEE Engineering in Medicine and Biology Society, pp. 3600-3603, Aug. 2006.

[13] Rougier, C. Meunier, J. St-Arnaud, A. Rousseau, J., "Monocular 3D Head Tracking to Detect Falls of Elderly People", In Proc. of 28th Annual International Conference of the IEEE Engineering in Medicine and Biology Society, pp. 6384 - 6387, Aug. 2006.

[14] Cao, X.B. Chen, D. Qiao, H. Xu, Y.W., "An Evolutionary Support Vector Machines Classifier for Pedestrian Detection", In Proc. of 2006 IEEE International Conference on Intelligent Robots and Systems, pp. 4223-4227, Oct. 2006.

[15] Jansen Bart, Deklerck Rudi, "Context aware inactivity recognition for visual fall detection", In Proc. of 2006 Pervasive Health Conference and Workshops, pp. 1-4, Dec. 2006.

[16] Gaura, E.I., Rider, R.J., Steele, N., Naguib, R.N.G., "Neural-network compensation methods for capacitive micromachined accelerometers for use in telecare medicine", IEEE Transactions on Information Technology in Biomedicine, Vol. 5, Issue 3., pp. 248-252, Sept. 2001.

[17] www.zigbee.org

[18] www.freescale.com

[19] Christianini N, Shawe-Taylor J: An introduction to support vector machines. Cambridge University Press, 2000.

[20] Schölkopf B: Statistical learning and kernel methods. [http://research.Microsoft.com/ bsc].

[21] Chih-Chung Chang and Chih-Jen Lin, LIBSVM : a library for support vector machines, 2001. Software available at http://www.csie.ntu.edu.tw/ cjlin/libsvm

[22] Sasiadek J.Z., Khe J., "Sensor fusion based on fuzzy Kalman filter", In proc. of 2nd International IEEE Workshop on Robot Motion and Control, pp. 275-283, Oct. 2001.

[23] Mohinder S. Grewal, Angus P. Andrews, Kalman Filterinf (Second Edition), John Wiley \& Sons, 2001.

[24] Virone G., Istrate D., Vacher M., Noury N., Serignat J.F., Demongeot J., "First Steps in Data Fusion between a Multichannel Audio Acquisition and an Information System for Home Healthcare", In Proc. of the $25^{\text {th }}$ Annual International Conference of the IEEE in Engineering in Medicine and Biology Society, vol 2., pp. 1364-1367, Sept. 2003

[25] Istrate D., Castelli E., Vacher M., Besacier L., Serignat J.-F., "Information extraction from sound for medical telemonitoring", IEEE Transaction on Information Theory in Biomedicine, vol. 10, 2, pp. 264274, April 2006.

[26] M Vacher, D Istrate, JF Serignat, "Sound Detection and Classification through transient models using Wavelet Coefficient Trees", presented at 12th Eurasip European Signal Processing Conference, Sept. 2004.

[27] Sonogram Speech and Sound processing tool, http://www.christophlauer.de/ 